Nachrichten der Deutschen Gesellschaft für Nuklearmedizin

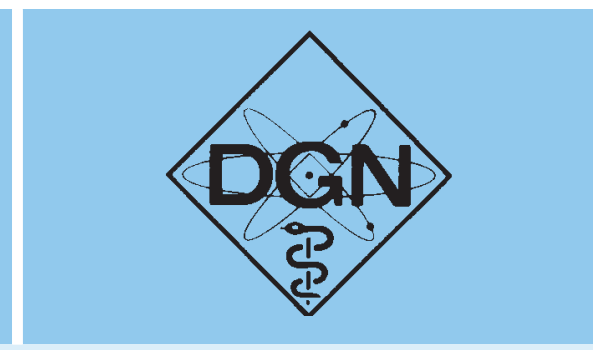

\title{
NuklearMedizin 2020 - Digital | Nachbetrachtungen
}

Unser Jahreskongress „NuklearMedizin 2020 - Digital“ liegt nun hinter uns! Die CoronaPandemie hat die Bedingungen vorgegeben. Unser für April in Leipzig geplantes Treffen wurde zunächst in den Juli verlegt, jedoch dann durch die Behörden untersagt und fand schließlich als Online-Kongress statt. Nur wenige Wochen Zeit standen zur kompletten Umstrukturierung und Planung zur Verfügung. Eine DGN-Taskforce wurde daher eingerichtet, die zusammen mit der DGN-Geschäftsstelle, dem Präsidium und der nmi GmbH das Heft in die Hand nahm. In der Rückschau ist besonders beachtlich, dass die schnell gefassten Pläne reibungslos umgesetzt wurden. Das wissenschaftliche Programm konnte in einer modifizierten Form durchgeführt werden. Dabei konnten auch die bereits im Jahr 2019 eingeführten neuen Formate zum großen Teil weitergeführt werden, lediglich auf den Zukunftskongress mussten wir verzichten. In den 7 Leuchtturm-Sitzungen wurden ausgewählte herausragende Beiträge präsentiert, diskutiert und kommentiert. Viele Beiträge wurden als e-Poster ohne Redebeitrag präsentiert. Das Fortbildungsprogramm wurde nahezu unverändert online durchgeführt. Auch das Post-Congress-Symposium, das sich in diesem Jahr unter dem Titel „Innovation auf dem Prüfstand“ mit der Weiterentwicklung unserer Instrumentierung beschäftigte, wurde online durchgeführt.

Zudem fanden 9 Industrie-Symposien statt; dies zeigt die weiterhin großartige Unterstützung der Industrie. Hierfür sei auch an dieser Stelle nochmals gedankt.

Wenig überraschend ist, dass unsere Teilnehmerzahl unter derjenigen des vergangenen Jahres lag. Jedoch scheint es angesichts der Umstände (inkl. Sommerferienbeginn in einigen Bundesländern) ein großer Erfolg, dass 927 Ärzte, MTRAs, Chemiker und Physiker an der Veranstaltung teilnahmen, pro Veranstaltung waren es im Mittel 136 Personen. Ein stattliches Ergebnis! Das Post-Congress-Symposium hatte im Vergleich zu den bisherigen Vorkongress-Symposien mit 305 Teilnehmern sogar die höchste Teilnehmerzahl aller Jahre. Die hohe Beteiligung an den TED-Umfragen und die zahlreichen Live-Anfragen im Chat zeigen, dass unsere Diskussionen lebhaft und fruchtbar verliefen und die aktuellen Themen in der Nuklearmedizin aufgegriffen wurden.

Vier Hauptthemen durchzogen den Kongress: die Digitalisierung der nuklearmedizinischen Diagnostik, die rechtlichen Grundlagen der Produktion von Radiopharmaka, die Gestaltung stationärer theranostischer Abteilungen sowie die Weiterentwicklung der Instrumentierung. Die Entwicklung unseres Fachgebietes wird in den nächsten Jahren maßgeblich von genau diesen Themen abhängen! Die Weiterführung der Diskussion dieser Fragen war deshalb dringend erforderlich und rechtfertigt die Durchführung des Kongresses im Online-Format.

Natürlich ersetzt das 2020 aufgrund der äußeren Umstände gewählte Format nicht einen Präsenzkongress. Die persönliche Begegnung ist nach wie vor ein entscheidender Aspekt unserer jährlichen Treffen. Wir sind zuversichtlich, dass wir uns in der Zukunft wieder auf Präsenzveranstaltungen freuen dürfen. Trotzdem sind wir überzeugt, dass Online-Formate weiter bestehen bleiben dürften und manche Veranstaltungen ergänzen oder sogar ersetzen werden. Vielleicht haben wir ja in der Online-Veranstaltung ein neues Hilfsmittel gewonnen, das es erlaubt, zügig kleinere Treffen zu ermöglichen und unsere Anliegen im Vergleich zu den bisher üblichen Telefonkonferenzen effektiver zu besprechen.

Es wird zukünftig eine weitere Änderung bei unseren DGN-Kongressen geben: Im Jahr 2021 wird erstmalig ein neuer Vorstand nach unserer neuen Satzung gewählt. Diese sieht keinen Kongresspräsidenten mehr vor. Vielmehr wird ein Mitglied des DGN-Vorstandes den Kongress als sogenannter Kongressvorstand verantwortlich organisieren. Markus Essler darf sich daher als letzter Kongresspräsident in der Geschichte der DGN von Ihnen verabschieden. Die Änderung ist zeitgemäß und notwendig. Nach einem Jahr voller Kongressvorbereitungen, Planungen und deren Änderung freuen wir uns, Euch und Sie auf dem 2021 hoffentlich als Präsenzmeeting stattfindenden DGN-Kongress in Leipzig wieder persönlich zu treffen, sofern die Pandemielage es zulässt. Bitte bleibt/bleiben Sie gesund!

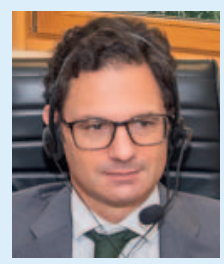

Prof. Dr. med. Markus Essler, Bonn

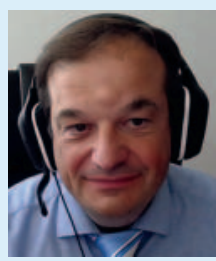

Prof. Dr. med. Michael Schäfers, Münster

VERANTWORTLICH FÜR DEN

INHALT

Deutsche Gesellschaft für

Nuklearmedizin e. V. (DGN)

Prof. Dr. Bernd Joachim Krause

(Präsident)

Nikolaistr. 29, 37073 Göttingen

E-Mail: office@nuklearmedizin.de

Schriftführer:

Prof. Dr. Peter Bartenstein

E-Mail: office@nuklearmedizin.de 